Informes de la Construcción Vol. 62, 520, 57-66, octubre-diciembre 2010 ISSN: 0020-0883 elSSN: 1988-3234 doi: 10.3989/ic.09.060

\title{
El tiempo equivalente de exposición al fuego en un edificio histórico
}

\author{
The equivalent time of fire exposure in a historical building
}

\section{B. Orta $^{(*)}$}

\section{RESUMEN}

Cuando se analiza la resistencia a fuego de una estructura, según el Código Técnico de Edificación (CTE), se puede recurrir a métodos simplificados que fijan valores de resistencias exigidas a la estructura, en función del uso y de la altura de evacuación del edificio, independientemente del sector, o bien se puede emplear el método del tiempo equivalente que permite considerar las condiciones particulares del sector estudiado (el tamaño, la ventilación, el material estructural, las medidas activas de lucha contra el fuego, etc.). En la mayor parte de los proyectos se optará por la primera vía, sin embargo cuando se trate de un edificio que por su singularidad requiera un análisis más pormenorizado, se recurrirá a la segunda.

Este artículo expone la dificultad de aplicar el método del tiempo equivalente en base al CTE sin consultar el Eurocódigo-1 (EC-1) o manuales técnicos. Se expone hasta dónde llega la norma española y cuando hay que acudir a la europea. Se aplica el método a un ejemplo concreto, un espacio en dos alturas con un hueco horizontal en cubierta. Además se muestra como el método del tiempo equivalente, como alternativa, puede generar una serie de resultados con una amplia dispersión de valores obtenidos a partir de la cantidad de decisiones que la normativa permite en función de unos criterios que no son rígidos. Esta diversidad de resultados puede dejar perplejo al calculista no docto en el asunto.

Para finalizar se extraen conclusiones sobre los valores obtenidos y se reflexiona sobre la aplicación del método y la responsabilidad del técnico.

Palabras clave: Estructuras de edificación, Tiempo equivalente, Resistencia a fuego, Código técnico de edificación, Seguridad contra incendios.

\section{SUMMARY}

When fire resistance of a structure is analysed, by means of Technical Building Code (CTE), it is possible to use simplified methods that fix values of resistances demanded from the structure, depending on use and building height of evacuation, independently of the sector, or it is possible to use the method of the equivalent Time that allows to consider the particular conditions of the studied sector (size, ventilation, structural material, active measures of fight against the fire, etc.). In most of projects the first route will be chosen, nevertheless when it is a question of a singular building that it needs a more detailed analysis, it will be appealed to the second one.

This article exposes the difficulty of applying the method of the equivalent time by means CTE without consulting the Eurocode-1 (EC-1) or technical manuals. It is exposed up to where the Spanish norm comes and when it is necessary to come to the European one. The method is applied to a specific example, a space in two heights by a horizontal hollow in cover. Also it shows as the method of the equivalent time, as alternative, can generate a series of results with a wide dispersion of values obtained from the quantity of decisions that the rules allows depending on a few criteria that are not rigid. This diversity of results can make not learned person who does calculations, perplex.

To finish conclusions on the obtained values are extracted and one thinks about the application of the method and the technician responsibility.

Keywords: Building structures, Equivalent time, Fire resistance, Technical Building Code, Security against fires. 
1. Solución estructural y constructiva del patio central (3).

2. Patio central.

\section{INTRODUCCIÓN}

Al estudiar la resistencia a fuego de una estructura según la normativa vigente en España, el Código Técnico de Edificación (CTE), se puede optar por el método tradicional, que fija valores de resistencias mínimas exigidas, o por cumplir el objetivo marcado mediante el método del tiempo equivalente de exposición al fuego.

En la mayor parte de los proyectos se tomarán los valores fijos tabulados en función de la altura de evacuación y el uso, sin embargo cuando se trate de un edificio que por su singularidad (en este caso por las características de catalogación del edificio) se requiera un análisis más pormenorizado se puede recurrir al cálculo del tiempo equivalente. Este cálculo no es inmediato, implica la toma de una serie de decisiones, en base a las alternativas de

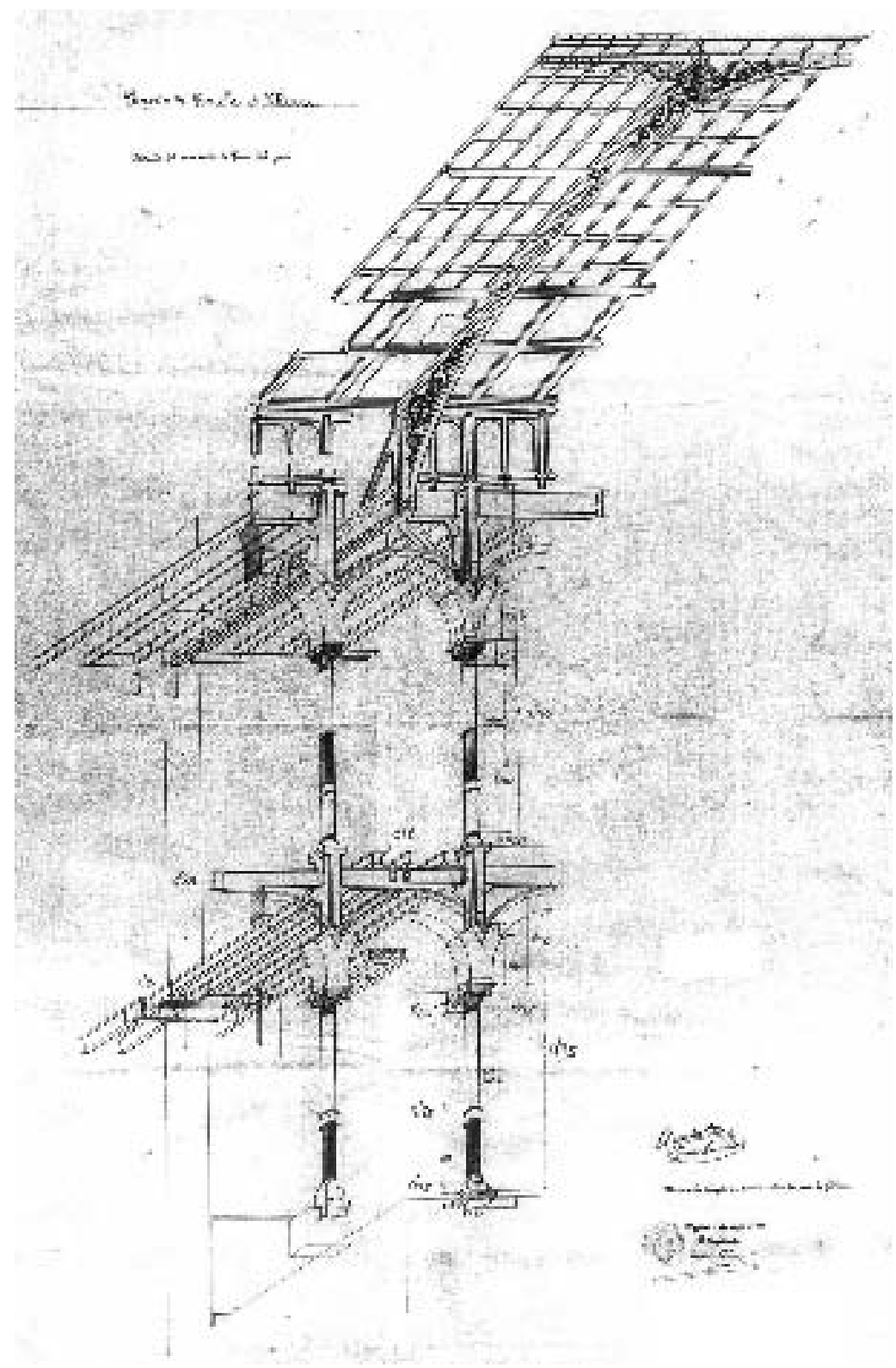

diseño particulares de cada caso, lo que puede producir una gran dispersión de resultados. En algunos aspectos es necesario recurrir al EC-1, tal y como refiere el propio CTE.

Se aplica el método a un ejemplo concreto, se muestran las diferentes alternativas de diseño posibles, y como influyen en las variables que intervienen en el cálculo, y así, en base a una serie de hipótesis, se obtienen diferentes valores de la magnitud buscada.

\section{DESCRIPCION DEL EDIFICIO}

Para este artículo se ha tomado como ejemplo el espacio más singular de un edificio histórico de gran valor en la arquitectura española y madrileña, el patio central de la Escuela de Ingenieros de Minas de Madrid. Se trata de un espacio que se desarrolla en dos alturas con arcadas sobre columnas de fundición, vistas, y una cubierta de acero y vidrio. La estructura principal es de muros de carga de ladrillo y elementos metálicos: columnas y vigas.

El edificio fue proyectado por Ricardo Velázquez Bosco en 1884 y fue construido entre los años 1886 y 1893 . Tiene planta rectangular, de 48 × 33 m, y consta de cuatro niveles: planta semisótano, plantas baja y primera que se desarrollan alrededor del patio central (Figuras 2, 3 y 4), y por último el nivel de torreones. El uso a que se destina actualmente es de carácter docente y administrativo.

El patio central, de $18 \times 10 \mathrm{~m}$, es el espacio más representativo. Está rodeado por arquerías sobre columnas de fundición vistas en dos niveles y su cubierta es de vidrio sobre una estructura de acero (Figuras 1, 2 y 3). Una serie de elementos decorativos acompaña tanto a elementos de cubierta como a barandillas del patio, columnas, etc.

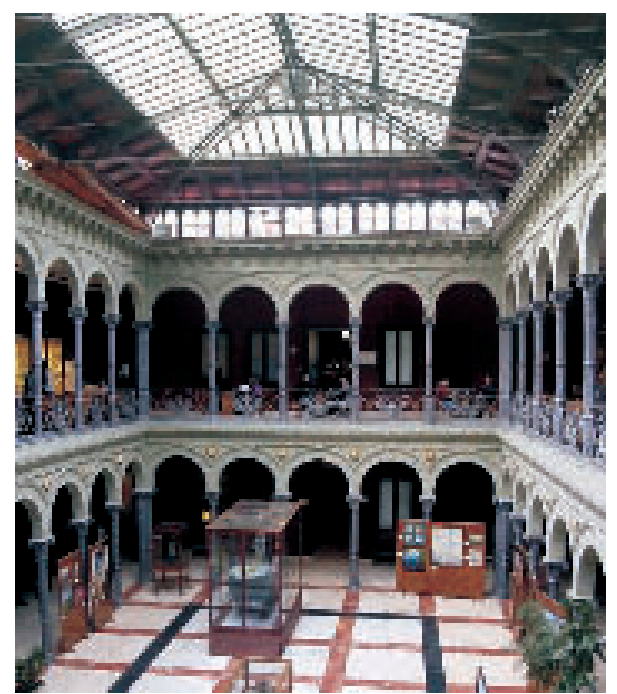


Al exterior destacan los elementos escultóricos de los torreones y la decoración de las fachadas cortas, ciegas, realizada por el ceramista Daniel Zuloaga. La fachada norte, correspondiente con la antigua aula de dibujo y actual biblioteca de alumnos, está formada por una columnata jónica de fundición con entrepaños acristalados.

La estructura vertical es, en su mayoría, de muros de carga realizada con fábrica de ladrillo que se combina con elementos de piedra de carácter decorativo. Alrededor del patio y en la fachada norte se disponen columnas de fundición vistas. Los forjados son de perfilería de acero (IPN de ala estrecha) y roscas de ladrillo in situ.

El edificio está considerado como un elemento relevante en la historia del Arte y la arquitectura española y madrileña, de ahí su catalogación y su grado de protección por la normativa urbanística de la ciudad. Esto implica que cualquier intervención que se acometa debe mantener sus características arquitectónicas y constructivas, volúmenes, formas y elementos decorativos.

\section{BREVE REVISION HISTORIA DEL MÉTODO DEL TIEMPO EQUIVALENTE}

A raíz de los trabajos de Ingberg (de 1921 a 1928), en el United States National Bureau of Standards, que estableció una relación directa entre la densidad de carga de fuego y la severidad del incendio para posteriormente proponer diferentes cargas de fuego para diferentes tipos de uso; y de los estudios de Kawagoe y Sekine (1958 a 1967), en el Building Research Institute de Tokio, en los que demostró el efecto de la ventilación en la severidad del incendio; Magnusson y Thelanderson desarrollan entre los años 1971 y 1972 una simulación numérica de las curvas tiempo-temperatura del proceso completo del desarrollo de un incendio en un recinto, se denominaron curvas de incendio real (8).

Pettersson, a partir de las curvas de Magnusson, propone que "una forma de tener el consideración la influencia de las características variables del desarrollo de un incendio para un cálculo diferenciado de estructuras resistentes al fuego, se basa en el concepto de tiempo equivalente de duración del incendio para hacer posible una relación directa entre las caracterización del incendio según las curvas de fuego real y las condiciones de calentamiento de los ensayos normalizados" (8).

El tiempo equivalente se obtiene a partir de la máxima temperatura, o mínima capacidad portante, obtenida para el elemento estructural en los análisis basados en la curva de fuego real, se transfiere a la curva de variación de la temperatura, o capacidad portante, del elemento según la curva de fuego normalizado y el tiempo obtenido es el valor buscado (Figura 5).
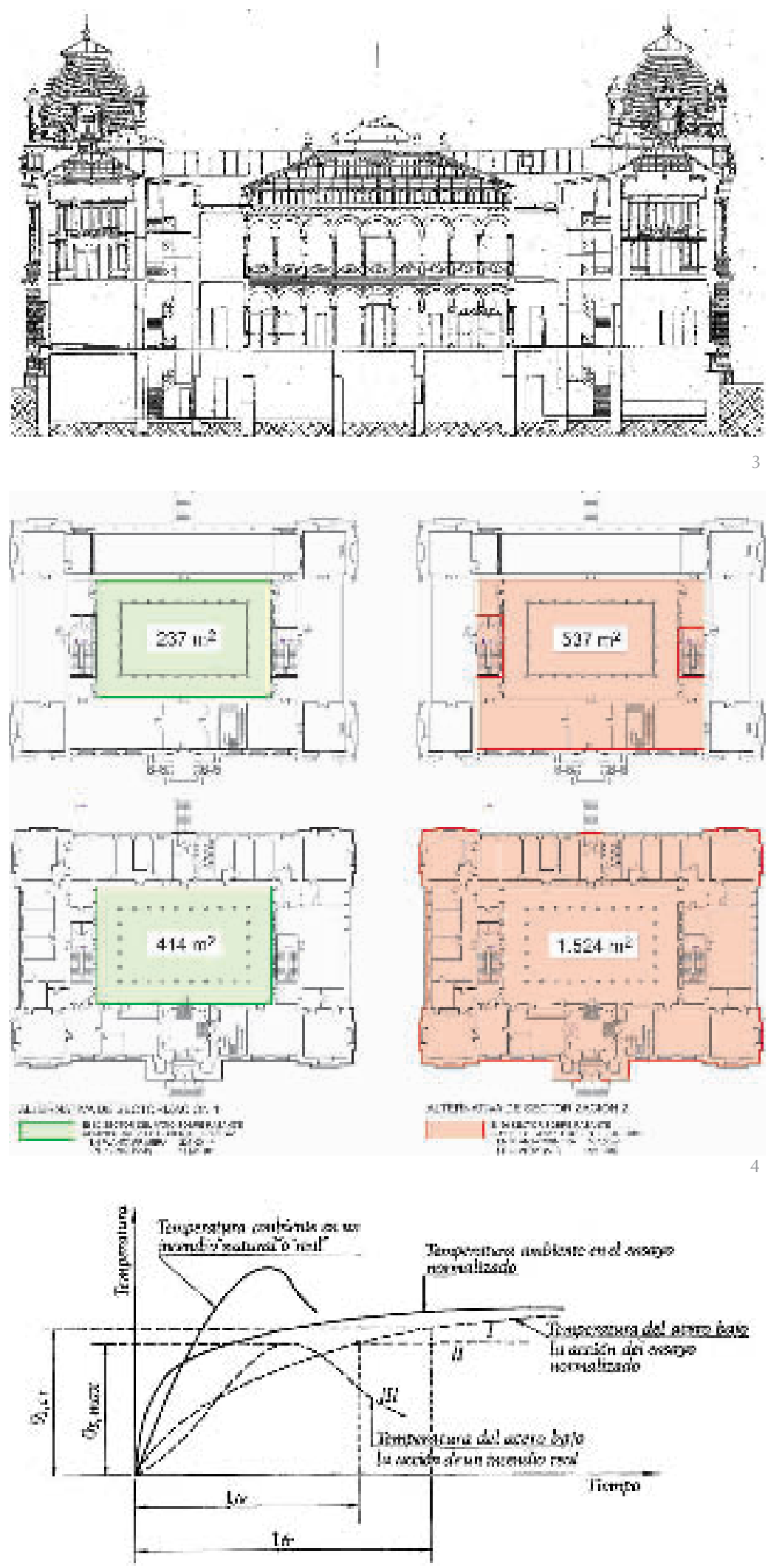

3. Sección longitudinal del edificio por el patio (3).

4. Sectorización alternativas 1 y 2, planta primera (arriba) y planta baja (abajo)

5. Definición clásica de tiempo equivalente (8). 
La aplicación de este modelo de análisis presenta el inconveniente de su extensión; como consecuencia numerosos investigadores, como Law o Thomas y Heselden (1972) dirigen sus trabajos a la elaboración de fórmulas que permitan la obtención más o menos exacta del tiempo equivalente de una estructura o elemento estructural. Pettersson propone una fórmula que recoge desde entonces gran numero de publicaciones, incluyendo el Comité Euro-International du Béton y European Recommendations. Se trata de una aproximación que depende solamente de las características del recinto, de la ventilación y la carga de fuego, siendo independiente de la estructura (8).

Posteriormente se incorporan modelos probabilistas que tienen en consideración factores de seguridad en el riesgo de vidas humanas, de daños materiales, incertidumbres debidas al uso de sistemas de detección y lucha contra el fuego, etc. incluyendo coeficientes de ponderación o reducción para la determinación del tiempo.

El Conseil International de Bâtiment M14 publica en 1983 una guía para el cálculo de la seguridad contra el fuego de las estructuras basada en conceptos de probabilidad, incluye el método del tiempo equivalente e incorpora factores de probabilidad mediante la introducción de coeficientes de ponderación para la carga combustible. El valor de estos coeficientes se encuentran en los actuales códigos nacionales e internacionales (8).

\section{PROCEDIMIENTO Y VARIABLES DE DISEÑO.}

Según el CTE (1) en su Documento Básico de Seguridad en caso de Incendio, DB-SI, la resistencia a fuego de una estructura es suficiente si a) alcanza la clase indicada en las tablas 3.1 y 3.2 del DB-SI-6 que representa el tiempo en minutos de resistencia ante esta acción, y se obtiene en función de la altura de evacuación y del uso del sector considerado; o si b) soporta la acción representada por la curva tiempotemperatura normalizada durante el tiempo equivalente de exposición al fuego, calculado según el anejo $B$ del mismo documento.

En este caso la altura de evacuación es menor a $15 \mathrm{~m}$ y el uso del sector es administrativo o docente, por lo que la clase resistente es R-60. Acudiendo a la curva normalizada tiempotemperatura se obtiene que en 60 minutos se alcanza una temperatura de $950^{\circ}$. Debido a que la transferencia de calor a la estructura sufre un retardo, se puede afirmar que la temperatura en la estructura puede estar entre $800^{\circ} \mathrm{C}$ y $900^{\circ} \mathrm{C}$, lo que implica una resistencia de la estructura del orden del $10 \%$ respecto al estado frío. Por lo tanto la estructura sin proteger no tiene la resistencia suficiente. Por otra parte debido a la catalogación del edificio no se puede proteger con los sistemas habituales sin afectar a las características arquitectónicas y elementos decorativos.

Por lo tanto se procede a calcular el Tiempo equivalente según el CTE (1), en los aspectos que no se encuentren resueltos en él se consultará el EC-1 (2), tal y como refiere el apartado de generalidades del DB-SI-6, así mismo se aclararán algunos aspectos con la bibliografía técnica $(4,5)$ y se consultará a expertos, siempre por este orden.

El valor del Tiempo equivalente depende de las características particulares del sector, se consideran aspectos como la forma y tamaño del sector de incendios, la existencia de huecos de ventilación y su tamaño, el material de la estructura, la instalación o no de medidas activas de lucha contra el fuego de manera voluntaria, el valor característico de la carga combustible, etc. Se plantean diferentes alternativas de diseño y posteriormente se combinarán en una serie de hipótesis para ver como influyen en valor final.

\subsection{Sectorización}

Se estudia el sector que incluye el patio central. Se contemplan dos alternativas; la primera incluye exclusivamente el área del patio y su pasillo perimetral en las dos alturas, son $237 \mathrm{~m}^{2}$ en planta primera y $414 \mathrm{~m}^{2}$ en planta baja, así la superficie de suelo del sector es $A_{f}=651 \mathrm{~m}^{2}$ (Figura 4 izquierda).

Por otra parte y dado que un sector de uso administrativo puede tener una superficie de hasta $2.500 \mathrm{~m}^{2}$, en la segunda alternativa se pretende alcanzar este valor; de esta manera el sector incluye toda la planta baja $\left(1.524 \mathrm{~m}^{2}\right)$ y algunas dependencias en planta alta $\left(537 \mathrm{~m}^{2}\right)$ con una superficie total de $A_{f}=2.061 \mathrm{~m}^{2}$ (Figura 4 derecha). No sólo cambian las superficies en planta, también lo hacen la superficie del contorno y los huecos verticales al exterior.

\subsection{Instalaciones}

Al analizar la evacuación del edificio en caso de incendio, el número de personas que lo hacen por el patio es menor que 500 por lo que no es obligatoria la instalación de detectores de humo. Se plantea por tanto la alternativa de disponer instalaciones de detección y extinción automáticos de incendio de manera voluntaria. Esta decisión influirá en el valor del tiempo equivalente a través del coeficiente que tiene en cuenta las medidas de protección activas existentes, $\delta_{n}$, uno de los factores que intervienen en la obtención 
del valor de calculo de la densidad de carga de fuego. Si no se instalan estas medidas el coeficiente valdrá uno $\left(\delta_{n}=1\right)$, y en caso de incluir la instalación de sistemas automáticos de detección $\left(\delta_{n 1}=0,87\right)$ y de extinción $\left(\delta_{n 3}=0,61\right)$ el coeficiente queda:

$$
\begin{gathered}
\delta_{n}=\delta_{n 1} \cdot \delta_{n 2} \cdot \delta_{n 3} \\
\delta_{n}=0,87 \cdot 1 \cdot 0,61=0,53
\end{gathered}
$$

\subsection{Ventilación}

El desarrollo de un incendio depende tanto de la aportación de oxígeno a través de las aberturas del recinto como de la evacuación del humos y calor. El coeficiente de ventilación, Wfi, así lo recoge, su valor varía con la alternativa de sectorización adoptada. En este aspecto se pretende estudiar la conveniencia de que la cubierta acristalada del patio esté abierta (cristales rotos al producirse el incendio) o cerrada (cristales resistentes al fuego). Cuando no hay cristales, más que tratarse de un edificio con un lucernario, sería como un edificio con un patio interior o un local sin techo (dado que la dimensión del lucernario es prácticamente igual a la dimensión del local), por lo tanto se analizaría como un espacio exterior, el humo y el calor producido en el incendio se evacuaría por el patio. Esta es por tanto la situación más favorable.

Por otra parte como los cristales no se rompen tan fácilmente, se debería estudiar la situación más desfavorable, analizando el espacio como interior, son los cristales sin romper. Probablemente la situación de incendio real no será ninguna de las dos, sino otra intermedia.

El coeficiente de ventilación se obtiene como indica el CTE según [2] con los significados indicados en el código. En este caso al ser un recinto de altura libre variable se toma el valor medio, por lo tanto $\mathrm{H}=12 \mathrm{~m}$.

$$
\begin{gathered}
\mathrm{W}_{\mathrm{f}}=(6 / H)^{0,3}\left[0,62+90\left(0,4-\alpha_{\mathrm{v}}\right)^{4 /}\right. \\
\left./\left(1+\mathrm{b}_{\mathrm{v}} \alpha_{\mathrm{h}}\right)\right] \geq 0,5
\end{gathered}
$$

Los valores obtenidos en las dos alternativas de sectorización (anotar que la sectorización 1 al ser solo el patio no tiene huecos verticales), y el lucernario cerrado $\left(A_{h}=0 \mathrm{~m}^{2}\right)$ son:

Tabla 1

Coeficiente de ventilación en función de las alternativas de diseño

\begin{tabular}{|l|r|r|r|c|c|c|}
\hline $\begin{array}{c}\text { Sectoriación/ } \\
\text { lucernario }\end{array}$ & $\begin{array}{c}\mathbf{A}_{\mathbf{v}} \\
{\left[\mathbf{m}^{2}\right]}\end{array}$ & $\begin{array}{c}\mathbf{A}_{\mathbf{f}} \\
{\left[\mathbf{m}^{2}\right]}\end{array}$ & $\begin{array}{c}\boldsymbol{\alpha}_{\mathbf{v}}= \\
\mathbf{A}_{\mathbf{v}} / \mathbf{A}_{\mathbf{f}}\end{array}$ & $\begin{array}{c}\mathbf{A}_{\mathbf{h}} \\
{\left[\mathbf{m}^{2}\right]}\end{array}$ & $\begin{array}{c}\boldsymbol{\alpha}_{\mathbf{h}}= \\
\mathbf{A}_{\mathbf{h}^{\prime}} / \mathbf{A}_{\mathbf{f}^{\prime}}\end{array}$ & $\mathbf{w}_{\mathbf{f}}$ \\
\hline 1/cerrado & 0 & 651 & 0 & 0 & 0 & $\mathbf{2 , 3 8}$ \\
\hline 2/cerrado & 306 & 2.061 & 0,15 & 0 & 0 & 0,80 \\
\hline
\end{tabular}

\subsection{Carga combustible}

La "carga combustible" o "carga de fuego", es la suma de las energías caloríficas que se liberan en la combustión de todos los materiales combustibles existentes en un espacio (elementos constructivos y contenidos del edificio), con excepción de aquellas materias que se encuentren en una solución acuosa o en un estado en el cual no son combustibles (4). El valor característico se obtiene de sumar el valor característico de la carga de fuego permanente (corresponde a los elementos constructivos combustibles incluidos los revestimientos y acabados permanentes del proyecto) y el valor variable. En este caso sólo hay el segundo término por lo que es el único que se calcula.

El valor característico de la densidad de carga combustible variable, por superficie de suelo, se puede determinar con los valores del CTE tabulados en función del uso. Al tratarse de dependencias dedicadas fundamentalmente a despachos, se considera del lado de la seguridad el valor correspondiente con uso administrativo, $520 \mathrm{MJ} / \mathrm{m}^{2}$, frente a $350 \mathrm{MJ} / \mathrm{m}^{2}$ de uso docente.

Paralelamente se determinará la carga combustible de forma específica elemento a elemento, como refiere el CTE (1), según el EC-1(2). Debido al poco mobiliario existente en el recinto del patio todo hace pensar que se obtendrá un valor menor. Para ello se obtiene la carga de fuego total por planta según [3] (Tabla 2 y Tabla 3):

$$
\mathrm{Q}_{\mathrm{fi}}=\sum \mathrm{M}_{\mathrm{i}} \cdot \mathrm{H}_{\mathrm{ui}} \cdot \psi_{\mathrm{i}}=\sum \mathrm{Q}_{\mathrm{fi}, \mathrm{h}, \mathrm{l}}
$$

donde:

Qfi carga de fuego total [MJ].

$M_{i}$ cantidad de material combustible [kg], obtenido de medir los elementos combustibles variables, y de la densidad del material (el mobiliario y toldos del lucernario).

$\mathrm{H}_{\text {ui }}$ potencial calorífico neto $[\mathrm{MJ} / \mathrm{kg}]$ valor tabulado en el EC-1 (2).

$\psi_{i} \quad$ coeficiente que permite evaluar las cargas de fuego protegidas, como en este caso no hay $\psi_{\mathrm{i}}=1$.

Tabla 2

Carga combustible en planta baja

\begin{tabular}{|c|c|c|c|c|}
\hline $\begin{array}{c}\mathbf{V} \\
{\left[\mathbf{m}^{3}\right]}\end{array}$ & $\begin{array}{c}\mathbf{\rho} \\
{\left[\mathbf{k g} / \mathbf{m}^{3}\right]}\end{array}$ & $\begin{array}{c}\mathbf{M}_{1} \\
{[\mathbf{k g}]}\end{array}$ & $\begin{array}{c}\mathbf{H}_{\mathrm{u} 1} \\
{[\mathbf{M} / \mathbf{k g}]}\end{array}$ & $\begin{array}{c}\mathbf{Q}_{\mathfrak{f} 1} \\
[\mathbf{M}]]\end{array}$ \\
\hline 2,34 & madera 500 & 1.170 & 17,5 & $\mathbf{2 0 . 5 0 0}$ \\
\hline
\end{tabular}

Tabla 3

Carga combustible en planta alta

\begin{tabular}{|c|c|c|c|c|}
\hline $\begin{array}{c}\mathbf{V} \\
{\left[\mathbf{m}^{3}\right]}\end{array}$ & $\begin{array}{c}\mathbf{\rho} \\
{\left[\mathbf{k g} / \mathbf{m}^{3}\right]}\end{array}$ & $\begin{array}{c}\mathbf{M}_{2} \\
{[\mathbf{k g}]}\end{array}$ & $\begin{array}{c}\mathbf{H}_{\mathbf{u} 2} \\
{[\mathbf{M J} / \mathbf{k g}]}\end{array}$ & $\begin{array}{c}\mathbf{Q}_{\mathfrak{f} 2} \\
{[\mathbf{M}]}\end{array}$ \\
\hline 7,50 & madera 500 & 3.750 & 17,5 & 65.600 \\
\hline- & telas & 145 & 20,0 & 2.900 \\
\hline \multicolumn{4}{|l}{} & $\mathbf{6 8 . 5 0 0}$ \\
\hline
\end{tabular}


Para obtener la densidad de carga característica relativa al área del suelo en este caso, con un sector de dos plantas, el EC-1 no indica como seguir, por lo que se procede según la bibliografía técnica $(4,5)$. Se calcula la carga combustible por unidad de superficie en planta en cada nivel, como se trata de carga de fuego variable, para obtener el valor característico se aplica [4] (Tabla 4):

$$
\mathrm{q}_{\mathrm{fi}, \mathrm{k}}=\mathrm{q}_{\mathrm{med}} \cdot\left(1+0,84 \mathrm{~V}_{\mathrm{q}}\right)
$$

donde:

$\mathrm{q}_{\mathrm{f}, \mathrm{k}}$ valor característico de la densidad de carga relativa al área del suelo por nivel.

$\mathrm{q}_{\text {med }}$ valor medio de la carga combustible obtenido a partir del valor estimado como probable en las tablas 2 y 3 por $\mathrm{q}_{\text {med }}=Q_{\mathrm{fi}} / \mathrm{A}_{\mathrm{i}}$, con:

$\mathrm{Q}_{\mathrm{fi}}$ suma de las cantidades de calor desarrollables por todos los materiales combustibles durante su combustión.

$A_{i} \quad$ superficie en planta del recinto al que corresponde la carga combustible.

$V_{q}$ coeficiente de dispersión unitaria, se toma el valor tabulado $\left(1+0,84 \cdot V_{\mathrm{q}}\right)$ en función del uso (1,25 en oficinas).

Tabla 4

Carga combustible característica por unidad de superficie en planta en cada nivel

\begin{tabular}{|c|c|c|c|c|}
\hline nivel & $\begin{array}{c}\mathrm{Q}_{\mathrm{fi}} \\
{[\mathrm{MJ}]}\end{array}$ & $\begin{array}{c}\mathbf{A}_{\mathbf{i}} \\
{\left[\mathbf{m}^{2}\right]}\end{array}$ & $\begin{array}{c}\left(1+0,84 \cdot V_{\mathrm{q}}\right) \\
\text { administra- } \\
\text { tivo } \\
\end{array}$ & $\begin{array}{c}\mathbf{q}_{\mathrm{fi}, \mathrm{k}} \\
{\left[\mathbf{M J} / \mathbf{m}^{2}\right]}\end{array}$ \\
\hline 11.0 & 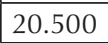 & 41 & 1,25 & 61, c \\
\hline prin & 68.500 & 237 & 1,25 & 36 \\
\hline
\end{tabular}

La carga combustible referida a la superficie total del contorno del recinto de incendio, al exceder de una planta del edificio se determina según [5] (Tabla 5):

$$
\mathrm{q}_{\mathrm{t}, \mathrm{k}}=\left(\sum \mathrm{A}_{\mathrm{i}} \cdot \mathrm{q}_{\mathrm{fi}, \mathrm{k}}\right) / \mathrm{A}_{\mathrm{t}}
$$

donde

$A_{t}$ superficie total de la envolvente del sector (paredes, suelo y techo) incluyendo aberturas y excluyendo los forjados intermedios.

Tabla 5

Carga combustible característica referida a la superficie total del contorno del recinto de incendio

\begin{tabular}{|l|c|c|c|c|}
\hline \multicolumn{1}{|c|}{ Nivel } & $\begin{array}{c}\mathbf{A}_{\mathbf{i}} \\
\mathbf{m}^{2}\end{array}$ & $\begin{array}{c}\mathbf{q}_{\mathrm{fi}, \mathbf{k}} \\
{\left[\mathbf{M} \mathbf{j} / \mathbf{m}^{2}\right]}\end{array}$ & $\begin{array}{c}\mathbf{A}_{\mathbf{t}} \\
\mathbf{m}^{2}\end{array}$ & $\begin{array}{c}\mathbf{q}_{\mathrm{t}, \mathbf{k}} \\
{\left[\mathbf{M} \mathbf{j} / \mathbf{m}^{2}\right]}\end{array}$ \\
\cline { 1 - 4 } pl baja & 414 & 61,90 & \multirow{2}{*}{1.689} & $\mathbf{6 5 , 8 8}$ \\
\cline { 1 - 3 } pl primera & 237 & 361,29 & & \\
\hline
\end{tabular}

Para pasarlo de la superficie de contorno a la superficie de suelo en proyección horizontal, $q_{i}$, hay que tomar una decisión sobre cómo considerar el área del suelo, $\mathrm{A}$; , se puede tomar el de todas las plantas o sólo el de planta baja, lo que da un valor más desfavorable y así se hace.

$$
q_{i k}=q_{t k} \cdot A_{t} / A_{f}
$$

\section{Tabla 6}

Carga combustible característica referida a la superficie del suelo del recinto de incendio

\begin{tabular}{|c|c|c|c|}
\hline $\begin{array}{c}\mathbf{q}_{\mathrm{tk}} \\
\left.[\mathbf{M}] / \mathbf{m}^{2}\right]\end{array}$ & $\begin{array}{c}\mathbf{A}_{\mathrm{t}} \\
{\left[\mathbf{m}^{2}\right]}\end{array}$ & $\begin{array}{c}\mathbf{A}_{\mathbf{f}} \\
{\left[\mathbf{m}^{2}\right]}\end{array}$ & $\begin{array}{c}\mathbf{q}_{\mathrm{fk}} \\
{\left[\mathbf{M} \mathbf{j} / \mathbf{m}^{2}\right]}\end{array}$ \\
\hline 65,88 & 1689 & 414 & $\mathbf{2 7 0}$ \\
\hline
\end{tabular}

El valor obtenido corresponde con la alternativa 1 de sectorización. En caso de la alternativa 2 no tiene sentido aplicar el mismo procedimiento, pues implicaría medir el mobiliario, el material de las oficinas incluido el papel, que pueden cambiar y de hacerlo así condicionaría el uso. En todo caso si se puede utilizar un procedimiento para calcular la carga combustible considerando que la zona del patio tiene una carga de $270 \mathrm{MJ} / \mathrm{m}^{2}$, valor real calculado, y el resto el valor tabulado correspondiente a uso administrativo, $520 \mathrm{MJ} / \mathrm{m}^{2}$ (Figura 6). Lo que se pretende es obtener un valor medio referido al área del suelo según [7] (Tabla 7).

$$
\mathrm{q}_{\mathrm{f}}=\sum \mathrm{q}_{\mathrm{i}} \cdot \mathrm{A}_{\mathrm{fi}} / \mathrm{A}_{\mathrm{f}}
$$

Tabla 7

\begin{tabular}{|c|c|c|c|c|}
\hline $\begin{array}{c}A_{\mathrm{fi}} \\
{\left[\mathrm{m}^{2}\right]}\end{array}$ & $\begin{array}{c}q_{i} \\
{\left[M J / m^{2}\right]}\end{array}$ & localización & $\begin{array}{c}A_{f} \\
{\left[\mathbf{m}^{2}\right]}\end{array}$ & $\begin{array}{c}\mathbf{q}_{\mathrm{f}} \\
{\left[\mathrm{MJ} / \mathrm{m}^{2}\right]}\end{array}$ \\
\hline 414 & 270 & PI baja patio & \multirow{4}{*}{2.061} & \multirow{4}{*}{441} \\
\hline 1.110 & 520 & PI baja resto & & \\
\hline 237 & 270 & PI primera patio & & \\
\hline 300 & 520 & $\mathrm{Pl}$ primera resto & & \\
\hline
\end{tabular}

Valor medio de la carga combustible referida a la superficie del suelo del recinto de incendio, método mixto

\subsection{Coeficiente de corrección según el material}

Se trata de una estructura de pilares de fundición y vigas de acero. Al tratarse de un edificio histórico no se puede proteger la estructura con los medios habituales sin afectar a las características arquitectónicas. Para el coeficiente de corrección en función del material, $\mathrm{k}_{\mathrm{c}}$ se considera el más similar, el acero sin proteger, por lo que su valor es $13,7 \cdot \mathrm{O}$, siendo $\mathrm{O}$ el coeficiente de aberturas y se obtiene a partir de:

$$
\mathrm{O}=\mathrm{A}_{\mathrm{v}} \sqrt{\mathrm{h}} / \mathrm{A}_{\mathrm{t}} .
$$




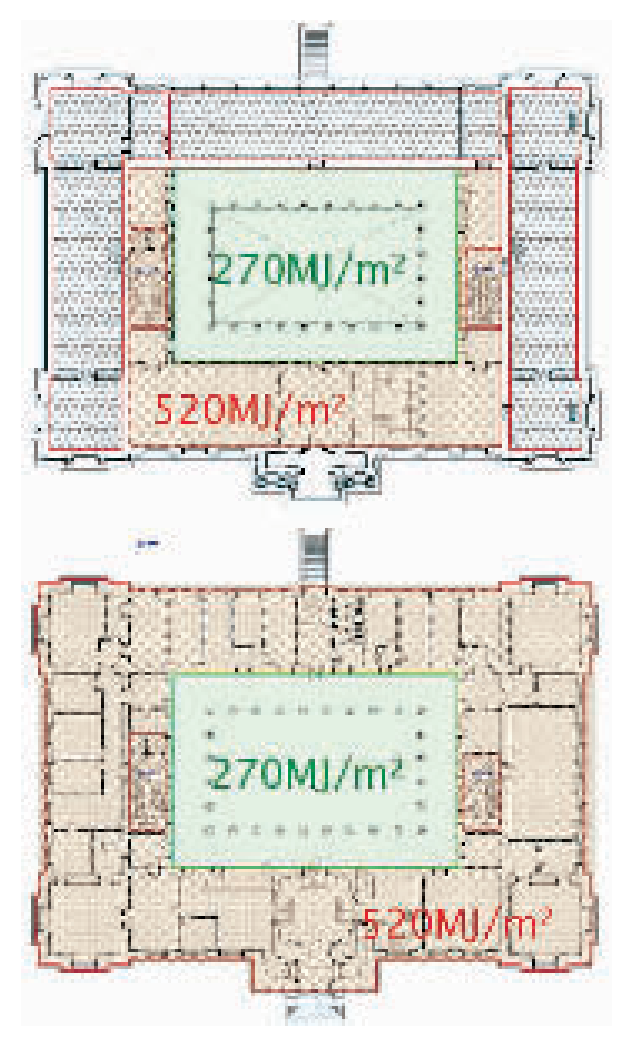

6

Tanto CTE como EC-1 no indican como se obtiene la altura promedio de los huecos verticales, $h$, se ha procedido en función de sus respectivas áreas $h=\left(\sum \mathrm{A}_{\mathrm{vi}} \cdot \mathrm{h}_{\mathrm{i}}\right) / \mathrm{A}_{\mathrm{v}}(4,5)$, con $A_{v i}$ superficie de la ventana $i, h_{i}$ altura de la ventana i, y $A_{v}$ superficie total de las aberturas verticales existentes en todas las paredes.

Tabla 8

Valor medio de la carga combustible referida a la superficie del suelo del recinto de incendio, método mixto

\begin{tabular}{|l|l|c|c|c|c|}
\hline & $\begin{array}{c}\mathbf{A}_{\mathbf{v}} \\
{\left[\mathbf{m}^{2}\right]}\end{array}$ & $\begin{array}{c}\mathbf{A}_{\mathbf{t}} \\
{\left[\mathbf{m}^{2}\right]}\end{array}$ & $\begin{array}{c}\mathbf{h} \\
{[\mathbf{m}]}\end{array}$ & $\begin{array}{c}\mathbf{O} \\
{\left[\mathbf{m}^{\mathbf{1} 2}\right]}\end{array}$ & $\begin{array}{c}\mathbf{k}_{\mathbf{c}} \\
-\end{array}$ \\
\hline 1/cerrado & 0 & 1689 & 0 & 0,02 & $\mathbf{0 , 2 7}$ \\
\hline 2/cerrado & 306 & 4586 & 2,85 & 0,112 & $\mathbf{1 , 5 4}$ \\
\hline
\end{tabular}

\section{TIEMPO EQUIVALENTE DE EXPOSICIÓN AL FUEGO: CÁLCULO}

El Tiempo equivalente de exposición al fuego se define en el CTE (1) y en el EC-1 (2) como "el tiempo de exposición a la curva normalizada tiempo-temperatura que se supone que tiene un efecto térmico igual al de un incendio real en el sector de incendio considerado".

El tiempo equivalente es el tiempo de ensayo normalizado en que un elemento alcanza una perdida de capacidad resistente como la máxima que sucede en el incendio real estudiado. Su valor se puede obtener a partir de la siguiente expresión [9] con los significados indicados en el CTE (1). Para el coeficiente de conversión en función de las propiedades térmicas de la envolvente del sector, kb; se ha tomado en todos los casos igual a 0,07, por defecto el valor máximo aunque se puede ajustar con los valores tabulados de (4).

$$
t_{e, d}=k_{b} \cdot w_{f} \cdot k_{c} \cdot q_{f, d}
$$

Los coeficientes de ventilación, $w_{f}$ y de corrección según el material estructural, $k_{c}$, se han calculado en los apartados 4.3 y 4.5 respectivamente. El valor de cálculo de la densidad de carga de fuego, $\mathrm{q}_{\mathrm{f}, \mathrm{d} /}$ en $\mathrm{MJ} / \mathrm{m}^{2}$, se determina a continuación.

\subsection{Densidad de carga combustible de cálculo}

La densidad de carga combustible de cálculo, $q_{\mathrm{f}, \mathrm{d}}$ se obtiene según [10] a partir del valor característico, $\mathrm{q}_{\mathrm{f}, \mathrm{k},}$ y de diversos factores $\left(\mathrm{m}, \delta_{\mathrm{q} 1}, \delta_{\mathrm{q} 2}, \delta_{\mathrm{n}}\right)$ que ponderan la repercusión estimada de cada unidad de carga combustible, según condiciones tales como la fracción de la misma que realmente arde en el incendio, y el riesgo de inicio de éste según el tamaño del sector, y según el tipo de actividad y la existencia de diferentes medidas activas de lucha contra el fuego. Un factor adicional $\delta_{\mathrm{c}}$ introduce aspectos de seguridad (4).

$$
\mathrm{q}_{\mathrm{fd}}=\mathrm{q}_{\mathrm{fi}} \cdot \mathrm{m} \cdot \delta_{\mathrm{q} 1} \cdot \delta_{\mathrm{q} 2} \cdot \delta_{\mathrm{n}} \cdot \delta_{\mathrm{c}}
$$

El CTE(1) indica que el coeficiente de combustión, m, en caso de madera y tejidos se puede tomar 0,8. Otra bibliografía (4) tabula este coeficiente pudiendo alcanzar el valor de 1,4 para madera o tejidos sueltos, hacerlo así aumentaría el tiempo equivalente. Como la madera quema muy bien parece recomendable entonces utilizar 0,8 cuando se emplea la densidad de carga combustible tabulada y usar $\mathrm{m}=1,4$ con la densidad de carga combustible calculada a mano.

El coeficiente que tiene en cuenta el riesgo de iniciación debido al tamaño del sector, $\delta_{\mathrm{q} 1}$, se interpola los valores del CTE siendo $\delta_{\mathrm{q} 1}=1,60$ para la primera alternativa de sectorización con $A_{f}=651 \mathrm{~m}^{2}$ y $\delta_{\mathrm{q} 1}=1,90$ para la segunda con $A_{f}=2.061 \mathrm{~m}^{2}$.

El coeficiente que tiene en cuenta el riesgo de iniciación debido al tipo de uso o actividad; en este caso uso administrativo o docente $\delta_{\mathrm{q} 2}=1$.

El coeficiente que tiene en cuenta las medidas activas voluntarias existentes, si no se instalan estas medidas $\delta_{n}=1$, en caso de incluir la
5. Densidad de carga de fuego por zonas. 
instalación de sistemas automáticos de detección y extinción $\delta_{\mathrm{n}}=0,53$ (apartado 4.2).

El coeficiente de corrección según las consecuencias del incendio, depende de la altura de evacuación del edificio, en este caso menor a $15 \mathrm{~m}$ por lo tanto $\delta_{\mathrm{c}}=1$.

\subsection{Hipótesis}

A continuación se obtiene la densidad de carga de fuego de cálculo (Tabla 9) y el tiempo equivalente (Tabla 10) en base a las alternativas de diseño combinadas en las siguientes hipótesis.

En la primera combinación se obtiene el tiempo equivalente a partir de la carga combustible real evaluada específicamente en 4.4, considerando el sector de incendio de la alternativa 1 (solo el patio), y no se disponen medidas activas voluntarias de lucha contra el fuego.

La segunda hipótesis se establece a partir de la anterior incluyendo medidas activas de lucha contra el fuego: sistemas automáticos de detección y extinción.
En la hipótesis tercera el valor característico de la densidad de carga de fuego es el tabulado para uso administrativo $\left(520 \mathrm{MJ} / \mathrm{m}^{2}\right)$, con la alternativa de sectorización 1 , se incluye la instalación de sistemas automáticos de detección y extinción de incendio.

Utilizando la sectorización 2 se establece la hipótesis cuarta, donde se considera además la instalación de sistemas automáticos de detección y extinción.

Las hipótesis quinta y sexta son similares a la anterior salvo que en la quinta no se emplean medidas activas de protección, por lo que $\delta_{n}=1$, y en la sexta la densidad de carga combustible que se calcula de manera mixta.

\section{ANALISIS DE RESULTADOS}

Analizando los valores obtenidos, que varían entre 15 y 68 minutos (Tabla 10), y teniendo como referencia el valor tabulado en el CTE en función de la altura de evacuación y del uso (60 minutos), se observa que todos los valores hallados son menores salvo en la hipótesis 5 con 68 minutos.

\section{Tabla 9}

Densidad de carga combustible, valor de cálculo en las diferentes hipótesis

\begin{tabular}{|c|c|c|c|c|c|c|c|}
\hline HIPÓTESIS & $\begin{array}{c}\mathbf{q}_{\mathbf{f k}} \\
{\left[\mathbf{M} \mathbf{j} / \mathbf{m}^{2}\right]}\end{array}$ & $\begin{array}{c}\mathbf{m} \\
{[\mathbf{m}]}\end{array}$ & $\begin{array}{c}\boldsymbol{\delta}_{\mathbf{q} 1} \\
-\end{array}$ & $\begin{array}{c}\boldsymbol{\delta}_{\mathbf{q} 2} \\
-\end{array}$ & $\begin{array}{c}\boldsymbol{\delta}_{\mathbf{n}} \\
-\end{array}$ & $\begin{array}{c}\boldsymbol{\delta}_{\mathbf{c}} \\
-\end{array}$ & $\begin{array}{c}\mathbf{q}_{\mathbf{f d}} \\
{\left[\mathbf{M} \mathbf{j} / \mathbf{m}^{2}\right]}\end{array}$ \\
\hline 1 & 270 & 1,4 & 1,6 & 1 & 1 & 1 & $\mathbf{6 0 5}$ \\
\hline 2 & 270 & 1,4 & 1,6 & 1 & 0,53 & 1 & $\mathbf{3 2 1}$ \\
\hline 3 & 520 & 0,8 & 1,6 & 1 & 0,53 & 1 & $\mathbf{3 5 3}$ \\
\hline 4 & 520 & 0,8 & 1,9 & 1 & 0,53 & 1 & $\mathbf{4 1 9}$ \\
\hline 5 & 520 & 0,8 & 1,9 & 1 & 1 & 1 & $\mathbf{7 9 0}$ \\
\hline 6 & 441 & 0,8 & 1,9 & 1 & 0,53 & 1 & $\mathbf{3 5 5}$ \\
\hline
\end{tabular}

Tabla 10

Tiempo equivalente de exposición al fuego de cálculo para las hipótesis planteadas

\begin{tabular}{|c|c|c|c|c|c|c|}
\hline \multicolumn{2}{|c|}{ HIPÓTESIS } & $\mathbf{k}_{\mathbf{b}}$ & $\mathbf{w}_{\mathbf{f}}$ & $\mathbf{k}_{\mathbf{c}}$ & $\mathbf{q}_{\mathrm{fd}}$ & $\mathbf{t}_{\mathrm{e}, \mathrm{d}}$ \\
\cline { 3 - 7 } $\mathbf{1}$ & {$[-]$} & {$[-]$} & {$[-]$} & {$\left[\mathbf{M} \mathbf{j} / \mathbf{m}^{2}\right]$} & {$[$ minutos] } \\
\hline $\mathbf{2}$ & $\begin{array}{c}\text { Sectorización 1 } \\
\text { sin instalaciones }\end{array}$ & 0,07 & 2,38 & 0,27 & 605 & $\mathbf{2 8}$ \\
\hline $\mathbf{3}$ & $\begin{array}{c}\mathrm{q}_{\mathrm{fik}} \text { real } \\
\text { Sectorización 1 } \\
\text { con instalaciones }\end{array}$ & 0,07 & 2,38 & 0,27 & 321 & $\mathbf{1 5}$ \\
\hline $\mathbf{4}$ & $\begin{array}{c}\mathrm{q}_{\mathrm{ik}} \text { tablas } \\
\text { Sectorización 1 } \\
\text { con instalaciones }\end{array}$ & 0,07 & 2,38 & 0,27 & 353 & $\mathbf{1 6}$ \\
\hline $\mathbf{5}$ & $\begin{array}{c}\mathrm{q}_{\mathrm{ik}} \text { tablas } \\
\text { Sectorización 2 } \\
\text { con instalaciones }\end{array}$ & 0,07 & 0,80 & 1,54 & 419 & $\mathbf{3 6}$ \\
\hline $\mathbf{6}$ & $\begin{array}{c}\mathrm{q}_{\mathrm{ik}} \text { tablas } \\
\text { Sectorización 2 } \\
\text { sin instalaciones }\end{array}$ & 0,07 & 0,80 & 1,54 & 790 & $\mathbf{6 8}$ \\
\hline $\begin{array}{c}\text { q }_{\mathrm{ik}} \text { mixta } \\
\text { Sectorización 2 } \\
\text { con instalaciones }\end{array}$ & 0,07 & 0,80 & 1,54 & 355 & 31 \\
\hline
\end{tabular}


En la hipótesis 1, al emplear una densidad de carga de fuego ajustada a la carga combustible real, aunque se toma el coeficiente de combustión máximo $m=1,4$, el tiempo equivalente es reducido (28 minutos), muy por debajo del valor de referencia.

Comparando la hipótesis 1 con la 2 se deduce que el uso simultaneo de sistemas automáticos de detección y extinción de incendios de manera voluntaria reduce el tiempo equivalente de manera proporcional al coeficiente encargado de su consideración, $\delta_{n}$, de ahí que sea aconsejable su instalación aunque no sea obligatorio. Lo mismo sucede al comparar las hipótesis $(4,5)$.

En este caso la carga combustible real es poca, con un valor de densidad característica de $270 \mathrm{MJ} / \mathrm{m}^{2}$, utilizado en la hipótesis 2, del orden de la mitad del indicado en la norma para uso administrativo $\left(520 \mathrm{MJ} / \mathrm{m}^{2}\right)$, usado en la hipótesis 3. Sin embargo, debido al coeficiente de combustión, los valores de cálculo se acercan siendo $321 \mathrm{MJ} / \mathrm{m}^{2}$ y $353 \mathrm{MJ} / \mathrm{m}^{2}$ respectivamente, el tiempo equivalente cambia proporcionalmente a este último. De manera similar sucede al comparar la hipótesis 4 con la 6 de nuevo cambia la densidad de carga de fuego y el tiempo equivalente proporcionalmente a ella.

La elección del tipo de sectorización influye de varias maneras, por una parte en función de su superficie se determina el coeficiente de riesgo de iniciación del incendio (con mayor superficie aumenta el coeficiente y el tiempo equivalente proporcionalmente); la superficie de su contorno influye en la disipación del calor por las paredes del sector (con mayor contorno el tiempo equivalente disminuye). El número y tamaño de huecos en fachada repercuten de dos modos, por una parte en la ventilación (con más huecos en fachada disminuye el coeficiente de ventilación y el tiempo equivalente de la misma manera) y por otra parte en el coeficiente de corrección del material por estar en función del coeficiente de aberturas (con más huecos crece el coeficiente y el tiempo equivalente). En este caso comparando la hipótesis 3 (sectorización 1) con la 4 (sectorización 2) la segunda alternativa es desfavorable. Es difícil establecer a priori la proporción con la que va a cambiar y como va a influir una sectorización u otra, al intervenir en varios aspectos, por lo que hay que estudiar cada propuesta de manera completa.

\section{CONCLUSIONES}

El método del tiempo equivalente se enmarca dentro de los métodos simplificados que emplean curvas de incendio normalizado (en las que la temperatura aumenta indefinidamente) frente a métodos más avanzados que emplean curvas de incendio real (en las que tras la fase inicial se incrementan fuertemente las temperaturas y al consumirse la carga combustible del sector la temperatura disminuye lentamente) o ensayos de resistencia real del elemento (procedimiento destructivo que en este caso no sería de aplicación). Es alternativo a las tablas de tiempos mínimos de resistencia exigidos a la estructura de un edificio, en función del uso y la altura de evacuación, que no permiten considerar las condiciones particulares del sector.

Como se ha visto su cálculo no es inmediato, implica tomar una serie de decisiones que influirán en el proyecto final. Para elegir las más adecuadas conviene conocer, en la etapa de diseño inicial, qué factores intervienen y como afectan al valor buscado. Es fácil conocer la influencia de algunas variables a priori (disponer sistemas activos de lucha contra el fuego siempre es favorable), en otros casos es más complejo (sectorización, aberturas verticales). La carga combustible se asocia al uso, se puede determinar mediante los valores tabulados en el CTE, preestablecidos por métodos estadísticos, o se puede obtener su valor elemento a elemento. La segunda vía implica una restricción en el uso que hay que controlar, no se podría añadir más carga combustible.

Es la primera vez que se incluye en la normativa española de seguridad a incendio el carácter prestacional a través del empleo del método del tiempo equivalente, que permite validar la solución propuesta sabiendo que se alcanzan los niveles de protección prefijados.

Por otra parte la norma no limita el campo de aplicación de este método. Con las hipótesis planteadas se obtienen tiempos entre 15 y 68 minutos, una amplia dispersión de valores a partir de la cantidad de decisiones tomadas que la normativa permite en función de unos criterios que no son rígidos. Esta diversidad de resultados puede dejar perplejo al calculista no docto en el asunto. Tanto el CTE como el EC-1 son normas que exponen el procedimiento a aplicar, en la actualidad hay escasez de bibliografía técnica que aporte una explicación del comportamiento físico del incendio y ayude a tomar una decisión.

La responsabilidad del técnico queda cubierta siempre que redacte un proyecto en base al CTE, también puede optar por otras alternativas mientras justifique adecuadamente su validez. Se podría plantear qué hacer cuando se obtienen valores mayores al de referencia, como el CTE no indica nada al respecto, todo parece apuntar a que tomar cualquiera de 
los valores obtenidos es aplicable a juicio del técnico mientras sea coherente con las decisiones tomadas en el cálculo.

Elegir entre las soluciones encontradas implica analizar las decisiones que hay detrás, unas son pura cuestión de cálculo y otras son económicas: la sectorización en espacios más pequeños (1-3) y el uso de medidas activas $(2-4,6)$ implican una mayor inversión económica en el proyecto (en puertas corta fuegos o instalaciones) si bien conducen a una economía en la protección de la estructura, representada en menores tiempos equivalentes que quizá permitirían dejar la estructura sin proteger.

En este caso hay que tener en cuenta no sólo el ahorro sino el interés histórico del edificio. Las alternativas con la sectorización 1 se descartan por requerir la compartimentación de espacios que en la actualidad no lo están, con el correspondiente perjuicio del patrimonio arquitectónico. Utilizando la sectorización 2 y sin instalar medidas activas (hipótesis 5) el tiempo obtenido es similar al de las tablas según uso; en este caso parece adecuado no utilizarla en el proyecto y se elegiría entre la 4 y 6 . La hipótesis 4 cumple la exigencia del CTE sin justificaciones mayores por utilizar valores del propio código por lo que parece la más adecuada.

El siguiente paso sería analizar la seguridad de los elementos bajo la acción del incendio normalizado correspondiente a un tiempo de 36 minutos, y conocer si para alcanzarlos es necesario la aplicación de algún tipo de material de protección de la estructura; de ser así y debido al interés histórico del edificio se optaría por una pintura intumescente para no desfigurar la imagen estructural del espacio.

\section{AGRADECIMIENTOS}

Quiero agradecer a Julia Villa Cellino (ETSAM) sus consejos como experta en el comportamiento de las estructuras ante el fuego y al personal de la ETSIM, José Ángel Sanchidrián (subdirector) y Feliciano Sánchez, haberme facilitado el acceso a sus dependencias.

\section{BIBLIOGRAFÍA}

(1) Código Técnico de la Edificación. Ministerio de Fomento 2004, www.codigotecnico.org

(2) UNE-EN 1991-1-2: Eurocódigo 1: Acciones en estructuras: Parte 1-2: Acciones de incendio. Bruselas, 2004.

(3) Archivo General de la Administración.

(4) Ortiz Herrera, J.; Villa Cellino, J.: Cálculo de las estructuras de acero frente a incendio, APTA, Madrid, 2009.

(5) Ortiz Herrera, J.; Villa Cellino, J.; Llamazares de la Puente, E.: Prontuario de ENSIDESA Tomo 1: La seguridad de las Estructuras de acero ante el incendio. Oviedo, 1989.

(6) Velazquez Bosco, R. (catalogo de Exposición dic 1990 feb-1991, MEAC): Ed. Dirección General de Bellas Artes y Archivos, Madrid, 1990, pp. 249-258.

(7) Villa Cellino, J.: La seguridad frente al incendio de las estructuras mixtas de edificación, vigas mixtas

(8) Llinares Cervera, M.; Maciá Torregrosa, M. E.; Arteaga Iriarte, A.: "Calibración del Tiempo Equivalente de Exposición al fuego según el Código Técnico de la Edificación", Informes de la Construcción, vol. 55 n. ${ }^{\circ} 490$ (2004). 